\title{
Prevention of the thrombosis that can be caused by COVID-19 vaccine
}

\section{Prevención de la trombosis que puede ser ocasionada por la vacuna contra COVID-19}

\author{
José L. García-Vigil1* and José L. García-Álvarez²
}

${ }^{1}$ Department of Pharmacology, Faculty of Medicine, Universidad Nacional Autónoma de México; ${ }^{2}$ Specialty Hospital, Centro Médico Nacional Siglo XXI, Instituto Mexicano del Seguro Social. Mexico City, Mexico

AstraZeneca's COVID-19 vaccine (Vaxzevria, formerly COVID-19 Vaccine AstraZeneca) prevents the disease caused by SARS-CoV-2 virus in people aged 18 years and older. The vaccine is composed of another virus from the adenovirus family that has been modified to contain a SARS-CoV-2 protein; since the vaccine does not contain the virus itself, it cannot cause COVID-19.

In different parts of the world, cases of venous and arterial thrombosis started to gradually be recorded in patients vaccinated with the aforementioned product, especially in the European Union, where its use was authorized by national regulatory agencies. This effect was regarded as an unusual situation that was not identified in the efficacy and safety clinical trials. For this reason, the European Medicines Agency (EMA) Safety Committee carried out an investigation on the subject and concluded that unusual blood clots with low platelet counts should be included as Vaxzevria's very rare side effects'. The EMA advises health-care professionals and people who receive the vaccine to be aware of the possibility of the occurrence of very rare cases of blood clots combined with low platelet counts, which are produced within 2 weeks after vaccination. So far, most reported cases have been documented in women younger than 60 years of age, with no specific risk factors having been identified. Out of 62 cases of cerebral venous sinus thrombosis and 24 of splanchnic venous thrombosis reported at the European Union drug safety database (EudraVigilance) by March 22, 2021, 18 were fatal.

In light of this information and available evidence, subject to having results from pharmacoeconomic studies such as cost-benefit, cost-effectiveness, and quality of life, the benefits so far outweigh the risks, and it is therefore advisable to continue with the vaccination plans in the different countries where the vaccine in question is being used, including Mexico. ${ }^{2}$ For this reason, prophylaxis with the use of antithrombotic drugs (antiplatelet agents and anticoagulants), already tested in both primary and secondary prevention, is justified, such as $100 \mathrm{mg} /$ day acetylsalicylic acid (aspirin) PO, $75 \mathrm{mg} /$ day dipyridamole PO, $2.5 \mathrm{mg} /$ day apixaban $\mathrm{PO}$, or $2.5 \mathrm{mg} /$ day rivaroxaban $\mathrm{PO}$, administered 1 week before the vaccination date and 2 weeks after. If necessary owing to pre-existing risk factors for hypercoagulability and under medical prescription, this prophylaxis could be continued in the long term.

\section{References}

1. European Medicine Agency. The Netherlands: AstraZeneca's COVID-19 Vaccine: EMA Finds Possible Link to Very Rare Cases of Unusual Blood Clots with low Blood Platelets. Amsterdam, Netherlands: European Medicine Agency; 2021.

2. World Health Organization. COVID-19: AstraZeneca Vaccine Benefits Still 'Largely Positive'. Geneva, Switzerland: World Health Organization; 2021 Apr 6. 